\title{
Deficient prefrontal attentional control in late-life generalized anxiety disorder: an fMRI investigation
}

\author{
RB Price ${ }^{1,2}$, DA Eldreth ${ }^{3}$ and J Mohlman ${ }^{1}$
}

Younger adults with anxiety disorders are known to show an attentional bias toward negative information. Little is known regarding the role of biased attention in anxious older adults, and even less is known about the neural substrates of any such bias. Functional magnetic resonance imaging (fMRI) was used to assess the mechanisms of attentional bias in late life by contrasting predictions of a top-down model emphasizing deficient prefrontal cortex (PFC) control and a bottom-up model emphasizing amygdalar hyperreactivity. In all, 16 older generalized anxiety disorder (GAD) patients (mean age $=66$ years) and 12 non-anxious controls (NACs; mean age $=67$ years) completed the emotional Stroop task to assess selective attention to negative words. Task-related fMRI data were concurrently acquired. Consistent with hypotheses, GAD participants were slower to identify the color of negative words relative to neutral, whereas NACs showed the opposite bias, responding more quickly to negative words. During negative words (in comparison with neutral), the NAC group showed PFC activations, coupled with deactivation of task-irrelevant emotional processing regions such as the amygdala and hippocampus. By contrast, GAD participants showed PFC decreases during negative words and no differences in amygdalar activity across word types. Across all participants, greater attentional bias toward negative words was correlated with decreased PFC recruitment. A significant positive correlation between attentional bias and amygdala activation was also present, but this relationship was mediated by PFC activity. These results are consistent with reduced prefrontal attentional control in late-life GAD. Strategies to enhance topdown attentional control may be particularly relevant in late-life GAD treatment.

Translational Psychiatry (2011) 1, e46; doi:10.1038/tp.2011.46; published online 4 October 2011

\section{Introduction}

In younger anxious individuals, attention is inordinately captured by negative cues, giving rise to an attentional bias. ${ }^{1-3}$ Despite the fact that anxiety disorders are prevalent in older adults, ${ }^{4}$ the impact of aging on attentional bias has received very little attention from researchers, with only 3 published studies ${ }^{5-7}$ as compared with $>200$ studies in younger adults. ${ }^{1}$ Older adults who are not assessed or selected for anxiety have been shown to differ from younger adults in their attention to emotional stimuli. In particular, when presented with positive, negative and neutral information, older adults are more likely than younger adults to allocate processing resources toward positive information, and away from negative. ${ }^{8,9}$ This 'positivity effect' could affect the attentional profile of older adults with clinical anxiety, warranting studies focused specifically on this age group.

Although in younger anxious samples, threat-related attentional bias is well established, there is no consensus regarding the cognitive mechanism that best accounts for the bias. ${ }^{1}$ Theoretical models differ in the extent to which they emphasize bottom-up processing, which is stimulus driven, rapid and typically occurs outside conscious volition and awareness, and top-down processing, which is driven by task demands and by consciously held goals, beliefs and strategies. Bottom-up and top-down models of bias imply dissociable neural substrates. For instance, an individual with a more reactive bottom-up system working to rapidly identify and orient toward threats ${ }^{10}$ would likely demonstrate hyperresponsivity of the amygdala, the principal brain region implicated in the processing of emotional material. ${ }^{11,12}$ Alternatively, an individual with a less efficient top-down executive system, who experiences difficulty implementing and enforcing processing routines relevant to task goals in the face of emotional distracters ${ }^{13}$ would likely show a pattern of deficient prefrontal cortex (PFC) recruitment. ${ }^{14}$ Given these dissociable neural profiles, neuroimaging studies may be uniquely well suited to clarify the model that best explains attentional bias in a given clinical population.

Few studies to date have examined the neural correlates of bias in clinically anxious samples. In non-clinical samples, the biased competition model of selective attention provides a relevant framework, suggesting an active interplay between bottom-up, sensory-driven mechanisms related to stimulus saliency and top-down mechanisms that implement selective attention through biasing signals. ${ }^{15}$ Pessoa et al. ${ }^{16}$ showed that this model is relevant to the processing of emotional stimuli and that bottom-up amygdalar responses are indeed subject to top-down control. These findings have been extended in studies of non-clinical samples designed to examine the relationship between anxiety (treated as a continuous variable) and brain activity during attentional bias

${ }^{1}$ Department of Psychology, Rutgers, The State University of New Jersey, New Brunswick, NJ, USA; ${ }^{2}$ Department of Psychiatry, University of Pittsburgh School of Medicine, Pittsburgh, PA, USA and ${ }^{3}$ Research Triangle Institute, Baltimore, MD, USA

Correspondence: Dr RB Price, Western Psychiatric Institute and Clinic, 3811 O'Hara Street, Pittsburgh, PA 15213, USA.

E-mail: rebecca.price@stanfordalumni.org

Keywords: aging; anxiety; attentional bias; attention; FMRI; prefrontal cortex

Received 6 June 2011; revised 5 September 2011; accepted 5 Septemeber 2011 
tasks. For instance, amygdala activity in response to threatrelated distracters has been shown to correlate positively with individual differences in self-reported 'state' anxiety during an attention bias task, whereas self-reported 'trait' anxiety was closely related to impoverished recruitment of top-down prefrontal control regions (lateral PFC, rostral anterior cingulate cortex (ACC)). ${ }^{17-19}$ On the basis of these and related findings, Bishop ${ }^{20,21}$ proposed an integrative neurocognitive account of anxious attentional bias, linking hyperresponsive, bottom-up amygdalar activity to state anxiety and deficient top-down PFC recruitment to trait anxiety. However, these findings have rarely been extended to the domain of treatment-seeking clinical populations.

Study aims. The purpose of this study was to explore the neural substrates of attentional bias in late-life generalized anxiety disorder (GAD), one of the most prevalent psychiatric conditions in older adults ${ }^{22}$ and a disorder reliably linked to attentional bias effects in younger samples. ${ }^{23}$ Treatment-seeking late-life GAD patients and an age-matched sample of non-anxious control (NAC) participants completed the emotional Stroop (eStroop) task while undergoing a functional magnetic resonance image (fMRI) scan. The GAD group was expected to exhibit longer color-identification reaction times (RTs) during presentation of negative words as compared with neutral, consistent with findings from younger GAD patients, whereas the NAC group was expected to show longer RTs during neutral word presentations (that is, a bias away from negative words), consistent with previous findings in non-anxious older adults. ${ }^{24} \mathrm{fMRI}$ analyses were designed to test the differing (although not mutually exclusive) predictions of contrasting models of eStroop interference: those emphasizing bottomup mechanisms, which predict amygdala hyperresponsivity to negative words in GAD, and those emphasizing top-down deficits in attentional control, which predict reduced recruitment of PFC regions (for example, ventrolateral, dorsolateral (DLPFC) and medial PFC, including the ACC) during negative words. Supplementary correlation and mediation analyses were designed to explore functional connections between the PFC and amygdala and their relation to behavioral performance. Although all reported findings were derived from whole-brain analyses, the current report focuses on these a priori regions relevant to bottom-up and top-down theoretical models (see Supplementary Information for a comprehensive list of significant clusters).

\section{Materials and methods}

Participants. Participants were 16 GAD and 12 NAC participants aged $\geqslant 60$ years who comprised a subsample of a larger group recruited for a study of cognitive-behavioral therapy for late-life GAD $(n=77) .{ }^{25}$ Study procedures were approved by the Institutional Review Board of the University of Medicine and Dentistry of New Jersey and Rutgers University. GAD participants were required to be free of any current comorbid axis I diagnosis and free of any major depressive episodes 'the past 5 years, as assessed by Structured Clinical Interview for DSM-IV. ${ }^{26}$ The non-anxious sample was required to be free of any lifetime axis I diagnosis (for additional clinical assessment and inclusion/exclusion details, see Supplementary Information). The groups were well matched on demographic variables and several self-reported health variables that may influence prefrontal function or the bloodoxygen-level-dependent signal (Table 1).

A combination of four neuropsychological tests was used as a dementia screen. All participants scored at least 25 on the Mini-Mental State Exam and achieved better than 'Borderline' (ninth percentile) scores on the Boston Naming Task ${ }^{27}$ and the Verbal Paired Associates and Similarities subtests of the WAIS. ${ }^{28}$ Participants also completed the 2-min Stroop ColorWord task, ${ }^{29}$ a test of non-emotional inhibitory control. Patients and controls did not differ on any neuropsychological measure (see Table 1).

eStroop task. The eStroop task ${ }^{30}$ is one of the most widely used paradigms in studies of attentional bias. The task requires participants to indicate the ink color of negative and neutral words as quickly as possible. Increased color-naming RTs during negative words are interpreted as an index of biased attention, suggesting that emotional information has captured processing resources despite its irrelevance to task performance. The limited late-life studies to date suggest that eStroop may elicit hypothesized biases in older adults more reliably than other widely used attentional bias tasks such as the dot probe ${ }^{5-7,31}$ perhaps because of its ability to capture interference flexibly on whatever time frame is suited to a participant's overall processing speed. ${ }^{6}$

The eStroop task was administered using a Dell desktop computer and E-Prime software (Psychology Software Tools,

Table 1 Descriptive statistics for generalized anxiety disorder and non-anxious control participants

\begin{tabular}{lcc}
\hline & $\begin{array}{c}\text { GAD group } \\
\mathbf{( n = 1 6 )}\end{array}$ & $\begin{array}{c}\text { NAC group } \\
\mathbf{( n = 1 2 )}\end{array}$ \\
\hline Age & $63.1(3.1)$ & $67.2(7.6)$ \\
Female & $11(69 \%)$ & $8(67 \%)$ \\
Caucasian & $14(88 \%)$ & $11(92 \%)$ \\
Education (years) & $16.2(2.9)$ & $16.9(2.8)$ \\
Household income & $\$ 46-60000$ & $\$ 46-60000$ \\
Age of GAD onset & $33.56(25.1)$ & - \\
Duration of illness & $34.75(25.2)$ & $-1.3(1.5)$ \\
Medications & $2.4(2.0)$ & $0.92(0.8)$ \\
Medical problems & $0.94(1.7)$ & $6.4(12.9)$ \\
Drinks per month & $7.7(12.7)$ & $0(0 \%)$ \\
Tobacco users & $0(0 \%)$ & $5(42 \%)$ \\
Hypertension & $7(44 \%)$ & $2(16 \%)$ \\
Diabetes & $1(6 \%)$ & $28.6(2.0)$ \\
MMSE & $28.3(1.3)$ & $55.2(8.5)$ \\
Boston naming task & $53.4(3.9)$ & $56.6(8.8)$ \\
Verbal paired associates & $52.4(9.9)$ & $59.8(4.5)$ \\
Similarities & $57.2(6.9)$ & $52.6(7.4)$ \\
Stroop color-word & $52.0(12.2)$ & $31.9(5.0) * \star *$ \\
PSWQ & $55.3(5.4)$ & $1.8(1.7)^{\star \star *}$ \\
GADQ-IV & $8.8(2.0)$ & $3.0(2.4)^{\star \star *}$ \\
BDI & $13.8(6.8)$ & \\
\hline & &
\end{tabular}

Abbreviations: BDI, Beck Depression Inventory; GAD, generalized anxiety disorder; GADQ-IV, Generalized Anxiety Disorder Questionnaire for DSM-IV; MMSE, Mini Mental State Exam; NAC, non-anxious control; Verbal Paired Associates and Similarities, subtasks of the Wechsler Adult Intelligence Scale, 3rd edition; PSWQ, Penn State Worry Questionnaire.

Neuropsychological measures presented as age-normed $t$-scores.

${ }^{\star * \star} P<0.001$ 
Sharpsburg, PA, USA; see Supplementary Information for additional information about the task and behavioral analyses). Using an approach validated previously in a larger sample of older adults, ${ }^{6}$ words were printed in red or green ink and participants responded by pressing a button with their right (red) or left (green) thumb. To maximize behavioral effect sizes, ${ }^{1}$ trials were presented as alternating blocks of negative and neutral words-16 words per block, 8 blocks in the experimental run. An equivalent number of negative and neutral words were presented in each color. Each trial began with a 1000-ms fixation crosshair, followed by a $2000 \mathrm{~ms}$ word presentation. For ease of interpretation, RT bias scores were calculated by subtracting each participant's mean RT for neutral words from his/her mean RT for negative words.

fMRI procedure. Images were acquired using a 3-T head-only Allegra scanner (Siemens Medical Systems, Ehrlangen, Germany) equipped with a fast gradient system for echoplanar imaging. Head motion was restricted using a standard radiofrequency head coil with foam padding. A threedimensional T1-weighted magnetization-prepared rapid gradient echo imaging sequence was used to acquire a highresolution anatomical scan for spatial normalization. Functional images were acquired using a $\mathrm{T}^{*}$-weighted echoplanar imaging sequence (repetition time $=2000 \mathrm{~ms}$, echo time $=30 \mathrm{~ms}$, flip angle $=90^{\circ}, 3.4 \times 3.4 \mathrm{~mm}^{2}$ in-plane resolution, 4-mm slice thickness, 32 axial slices). Data acquisition was preceded by $18 \mathrm{~s}$ of gradient and radiofrequency pulses to allow tissue to reach steady-state magnetization.

fMRI data analysis. Functional MRI data were processed offline using Statistical Parametric Mapping software (SPM5; Wellcome Department of Imaging Neuroscience, London, UK) and associated toolboxes. Functional volumes were slice time corrected and spatially realigned to correct for motion. Data sets that exhibited movement of $>3 \mathrm{mms}$ or $3^{\circ}$ of rotation $(n=2)$ were not included in the final sample described in Table 1. The magnetization-prepared rapid gradient echo imaging was linearly co-registered to the functional images. To compensate for heterogeneity of brain anatomy, which increases with age, SPM5's DARTEL toolbox was used to create a custom template and normalize functional and anatomical scans to Montreal Neurologic Institute space. ${ }^{32}$ The DARTEL algorithm performed favorably in a recent comparison of 14 nonlinear deformation algorithms for brain image registration, particularly when applied to a data set with a wide age range. ${ }^{33}$ Normalized functional images were spatially smoothed with an 8-mm fullwidth half-maximum Gaussian kernel.

A whole-brain general linear model approach was used to identify voxels the activity of which covaried in time with the emotional valence of eStroop stimuli. Although trials were presented in a block design, an event-related analysis was used to model the onset time of each trial as a distinct event. This approach may provide a more accurate model of the hemodynamic response and capture a greater degree of task-related variance in comparison with a conventional boxcar regressor approach for block designs. ${ }^{34}$ Consistent with an increase in power from this approach, whole-brain conventional block design analyses yielded a similar but slightly more constrained pattern of findings than those reported here. Task-related activity was modeled by convolving a vector of the trial onset times with a canonical hemodynamic response function. A 128-s high-pass filter and motion parameters were included as nuisance covariates. Negative $>$ neutral contrast maps were created for each individual at the single-subject level and then subjected to random effects group-level analysis, with each individual's average RT across all trials included as a nuisance covariate. Adding age, gender or Beck Depression Inventory (BDI) scores as additional covariates in group-level analyses did not have a substantial effect on the clusters observed in any analysis (see Supplementary Information).

Thresholds for multiple comparison correction were derived using AlphaSim (http://afni.nimh.nih.gov/pub/dist/doc/manual/AlphaSim.pdf), a Monte Carlo simulation program in the AFNI library. For a given voxel-wise probability threshold and a given search space (such as the whole brain or a subregion of interest), this method derives the cluster volume needed to hold the false-positive rate for cluster detection at a desired level. Using a voxel-wise threshold of $P<0.005$, a cluster volume threshold of 102 contiguous $2-\mathrm{mm}^{3}$ voxels was determined necessary to hold the probability of map-wise false-positive detection at $P<0.05$ in whole-brain analyses. Owing to an a priori interest in the amygdala, a small volume correction was applied to this region using an anatomical region of interest (ROI) mask for the left and right amygdalae created in SPM's Anatomy toolbox (http://www.fz-juelich.de/ inb/inb-3/spm_anatomy_toolbox). Using a voxel-wise threshold of $P<0.005$, a cluster volume threshold of 17 contiguous voxels was derived for the amygdala using AlphaSim.

Correlation and mediation analysis. Correlations across brain regions, and between brain regions and $\mathrm{RT}$ bias scores, were calculated as Pearson's $r$. The Multilevel Mediation and Moderation Toolbox (http://www.columbia.edu/cu/psychology/ tor/mediation.html) was used to conduct statistical tests of correlation coefficients and to perform single-level mediation analysis based on a standard three-variable path mode ${ }^{35}$ with an additional test for the indirect effect of the mediator (see Supplementary Information). In mediation analyses, given that sample sizes were small, hypotheses in these analyses were clearly directional, and a conservative definition of mediation was used, significance tests were one-tailed. All other reported $P$-values are two-tailed.

\section{Results}

Behavioral results. As hypothesized, the GAD group exhibited a positive RT bias score (mean=11.94 ms, s.d.=32.4), indicating greater interference from negative words than from neutral, whereas the NAC group exhibited a negative bias score (mean $=-10.91 \mathrm{~ms}$, s.d. $=20.6$ ), indicating increased task efficiency during negative items as compared with neutral (between-groups $t_{26}=2.14, P=0.04$ ).

\section{fMRI results}

Between-group results. Regions showing group differences in the neural response to negative vs neutral words are 
presented in Table 2. Consistent with a top-down deficits model, the NAC group exhibited larger negative vs neutral contrast values than did the GAD group in two PFC regions: the left DLPFC and the right rostral DLPFC (Figure 1).

Applying a small volume correction to the amygdala revealed a cluster in the left basolateral amygdala for which the GAD group showed greater negative vs neutral contrast values than did the NAC group (peak voxel $Z=3.10$, $P<0.001 ; n=18$; map-wise $P=0.04$ ).

Within-group results. Brain regions showing differential activity as a function of valence (negative vs neutral) are presented separately for each group in Table 3 . In the GAD group, no significant clusters were found exhibiting greater activity during negative than neutral words. Two PFC clusters exhibited decreased activity during negative words as compared with neutral: the right and left DLPFC (Figure 1).

In the NAC group, two PFC regions showed greater activity during negative words than neutral: the bilateral rostral DMPFC and left ventrolateral PFC (Figure 1). An emotional processing cluster encompassing portions of the left basolateral amygdala, hippocampus and temporal pole showed decreased activity during negative words as compared with neutral.

Applying a small volume correction to the amygdala did not reveal any new significant findings for any contrast, although this analysis confirmed that the amygdala/hippocampus cluster identified in the NAC group analysis above was significant when considering voxels in the amygdala only.

We conducted additional within-group analyses to explore whether the PFC findings revealed in between-groups analysis were better explained by PFC increases during negative words in the NAC group, PFC decreases during negative words in the GAD group or both. The search space for these analyses was restricted to functional ROls defined as all suprathreshold voxels in the left DLPFC and right rostral DLPFC clusters shown in Table 2, using cluster extent thresholds derived by AlphaSim to hold $P<0.05$ within the search space. These analyses revealed significant clusters within the left DLPFC ROI (peak voxel: $x=-54, y=22$,

Table 2 Between-group comparisons of BOLD signal for generalized anxiety disorder and non-anxious control participants during the negative-neutral run (negative $>$ neutral contrast)

\begin{tabular}{|c|c|c|c|c|c|c|c|}
\hline Region & $\begin{array}{l}\text { Location of } \\
\text { peak voxel }\end{array}$ & Brodmann's areas & $\mathbf{x}$ & $\mathbf{y}$ & $\mathbf{z}$ & $\begin{array}{c}\text { Cluster extent } \\
\text { (number of voxels) }\end{array}$ & Peak Z \\
\hline \multicolumn{8}{|l|}{$N A C>G A D$} \\
\hline $\begin{array}{l}\text { Dorsolateral PFC } \\
\text { Rostral dorsolateral PFC }\end{array}$ & $\begin{array}{l}\mathrm{L} \text { inferior frontal gyrus } \\
\mathrm{R} \text { superior frontal gyrus }\end{array}$ & $\begin{array}{l}45,46 \\
8,9\end{array}$ & $\begin{array}{r}-58 \\
22\end{array}$ & $\begin{array}{l}18 \\
38\end{array}$ & $\begin{array}{l}24 \\
44\end{array}$ & $\begin{array}{l}112 \\
113\end{array}$ & $\begin{array}{l}3.66 \\
3.27\end{array}$ \\
\hline $\begin{array}{l}G A D>N A C \\
\text { Amygdala (applying } \\
\text { anatomical mask) }\end{array}$ & L basolateral amygdala & & -24 & -2 & -26 & 18 & 3.10 \\
\hline
\end{tabular}

Abbreviations: BOLD, blood-oxygen-level-dependent; GAD, generalized anxiety disorder; NAC, non-anxious control; PFC, prefrontal cortex; SPM, Statistical Parametric Mapping.

Coordinates for peak voxels are presented in the Montreal Neurological Institute (MNI) space. Anatomical mask for the amygdala (left and right) created using SPM's Anatomy toolbox and small volume corrected at $P<0.05$. All other findings are from unrestricted whole-brain analysis with map-wise error rate $P<0.05$.
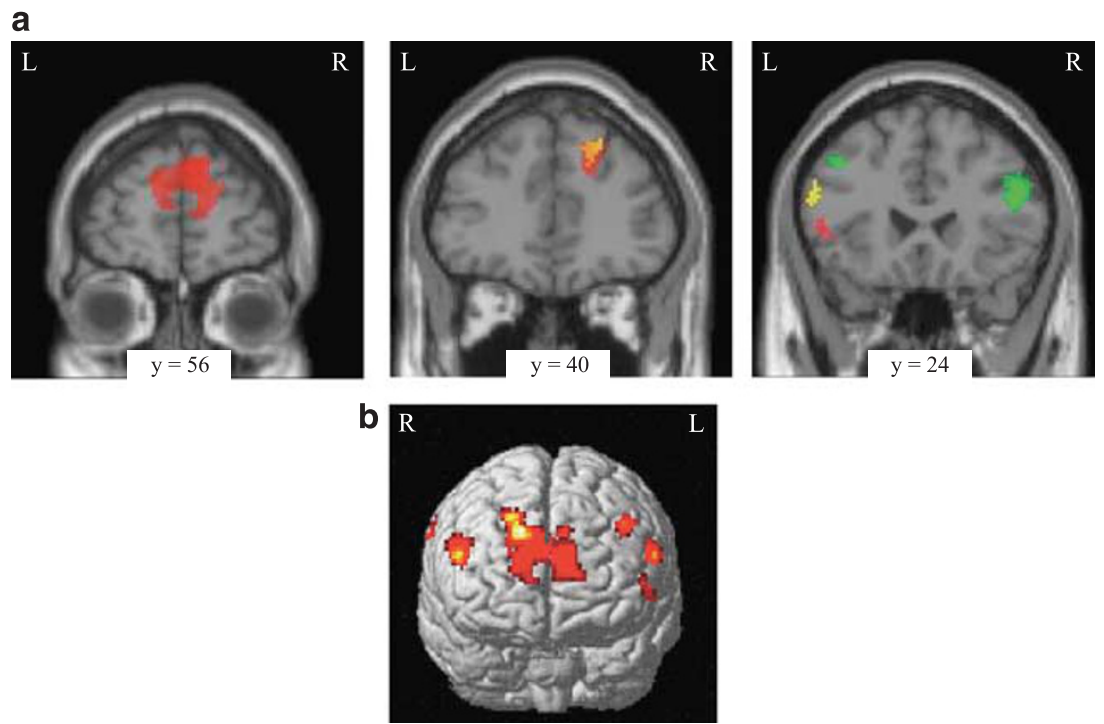

Figure 1 (a) Prefrontal cortex regions exhibiting greater activation to negative words than neutral in the NAC group (red), lesser activation to negative words than neutral in the GAD group (green) and greater negative > neutral contrast values in the NAC group than in the GAD group (yellow). Middle panel shows overlapping red and yellow clusters. Images are displayed in neurological convention (right = right). All voxels shown are significant at $P<0.005$, uncorrected and $P<0.05$, corrected. (b) Surface rendering of the regions shown in panel a. Image is shown in radiological convention (left= right). NAC, non-anxious control; GAD, generalized anxiety disorder. 
$\left.z=20 ; Z=3.02 ; n=26 ; P_{\text {corrected }}=0.002\right)$ and right rostral DLPFC ROI (peak voxel: $x=18, y=42, z=34 ; Z=4.12$; $\left.n=98 ; P_{\text {corrected }}<.0001\right)$ that exhibited greater activity during negative than neutral words in the NAC group, as well as a significant cluster within the left DLPFC ROI (peak voxel: $\left.x=-60, y=14, z=26 ; Z=3.35 ; n=20 ; P_{\text {corrected }}=0.004\right)$ that exhibited greater activity during neutral than negative words in the GAD group. Thus, although between-group differences in the right rostral DLPFC seem to be driven by increases in the NAC group, left DLPFC between-group findings can best be explained jointly by opposing patterns of PFC activity across the two groups.

Correlation and mediation analysis. Correlation analyses were used to test whether greater RT bias would be related to reduced activation of PFC regions and/or increased activation of the amygdala. On the basis of both within- and between-group findings, functional ROls were created for all clusters lying in the PFC (six clusters) or the amygdala (two clusters). The mean negative $>$ neutral contrast value across all supratheshhold voxels in a given cluster was extracted for each individual. Across all participants, RT bias scores were negatively correlated with contrast values in numerous PFC ROls, including the DMPFC ( $r=-0.44, P=0.016)$, the right $\operatorname{DLPFC}(r=-0.41, P=0.036)$ and the left DLPFC $(r=-0.46$, $P=0.002)$ ROls derived from within-group analyses, and the right rostral DLPFC $(r=-0.50, P=0.004)$ and left DLPFC ( $r=-0.43, \quad P=0.02$ ) ROls derived from between-group analyses. RT bias was positively correlated with contrast values from the NAC within-group left amygdala/ hippocampus ROI $(r=0.43, P=0.024)$ and marginally associated with the left amygdala ROI from betweengroups analysis ( $r=0.37, P=0.052)$.

Contrast values from two between-groups ROls also exhibited an inverse relationship with one another: the left amygdala and the right rostral DLPFC $(r=-0.45, P=0.024$; Figure 2). These three interrelated variables (right DLPFC values, left amygdala values and RT bias scores) were thus subjected to a mediation analysis designed to test the hypothesis that the relationship between greater amygdala activation and greater RT bias was mediated by the degree of DLPFC activation exhibited across individuals. Testing the path coefficients for a standard three-variable path model, significant relationships were observed between left amygdala activity and RT bias scores (path c: $\beta=0.38, P=0.026$ ), left amygdala activity and right DLPFC activity (path a: $\beta=-0.46$, $P=0.01)$ and right DLPFC activity and RT bias scores, controlling for left amygdala activity (path b: $\beta=-0.41$, $P=0.024)$. Furthermore, the relationship between left amygdala activity and RT bias was significantly reduced when right DLPFC values were included in the model (path $\mathrm{c}-\mathrm{c}^{\prime}$ : $\beta=0.18, P=0.034$ ), leaving the relationship between the amygdala and RT bias non-significant (path $\mathrm{c}^{\prime}: \beta=0.20$, $P=0.20)$. Thus, all four of the statistical criteria for mediation were met, indicating that across all participants, increased

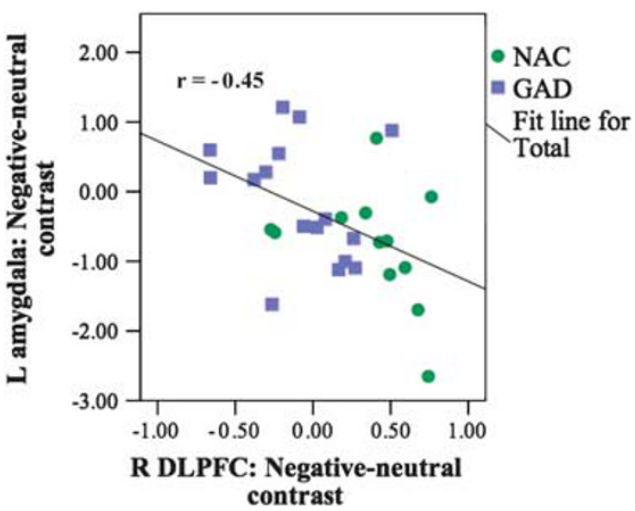

Figure 2 Scatterplot showing functional connectivity between left amygdala contrast values and right dorsolateral prefrontal cortex (DLPFC) contrast values. Contrast values are calculated for each individual as the difference between $\beta$-weights for regressors modeling the hemodynamic response to negative and neutral words, averaged across all voxels in each functional region of interest. NAC, non-anxious control; GAD, generalized anxiety disorder.

Table 3 Within-group comparisons of BOLD signal for generalized anxiety disorder and non-anxious control participants

\begin{tabular}{|c|c|c|c|c|c|c|c|}
\hline Region & $\begin{array}{l}\text { Location of } \\
\text { peak voxel }\end{array}$ & Brodmann's areas & $\mathbf{x}$ & $y$ & z & $\begin{array}{c}\text { Cluster extent } \\
\text { (number of voxels) }\end{array}$ & Peak Z \\
\hline $\begin{array}{l}\text { Negative }>\text { neutral } \\
\text { GAD group } \\
\text { No significant clusters } \\
\text { NAC group }\end{array}$ & & & & & & & \\
\hline $\begin{array}{l}\text { Dorsomedial PFC } \\
\text { Ventrolateral PFC }\end{array}$ & $\begin{array}{l}\mathrm{R} \text { superior frontal gyrus } \\
\mathrm{L} \text { inferior frontal gyrus }\end{array}$ & $\begin{array}{l}9,10 \\
45,47\end{array}$ & $\begin{array}{r}10 \\
-50\end{array}$ & $\begin{array}{l}54 \\
26\end{array}$ & $\begin{array}{r}32 \\
6\end{array}$ & $\begin{array}{l}941 \\
104\end{array}$ & $\begin{array}{l}4.25 \\
3.26\end{array}$ \\
\hline $\begin{array}{l}\text { Neutral > negative } \\
\text { GAD group }\end{array}$ & & & & & & & \\
\hline $\begin{array}{l}\text { Dorsolateral PFC } \\
\text { Dorsolateral PFC }\end{array}$ & $\begin{array}{l}\mathrm{R} \text { inferior frontal gyrus } \\
\mathrm{L} \text { middle frontal gyrus }\end{array}$ & $\begin{array}{c}45,46 \\
9\end{array}$ & $\begin{array}{r}48 \\
-40\end{array}$ & $\begin{array}{l}26 \\
28\end{array}$ & $\begin{array}{l}20 \\
40\end{array}$ & $\begin{array}{l}253 \\
103\end{array}$ & $\begin{array}{l}4.27 \\
3.70\end{array}$ \\
\hline $\begin{array}{l}\text { NAC group } \\
\text { Amygdala/hippocampus } \\
\text { Amygdala (applying } \\
\text { anatomical mask) }\end{array}$ & $\begin{array}{l}\mathrm{L} \text { basolateral amygdala } \\
\mathrm{L} \text { basolateral amygdala }\end{array}$ & & $\begin{array}{l}-24 \\
-24\end{array}$ & $\begin{array}{l}-2 \\
-2\end{array}$ & $\begin{array}{l}-26 \\
-26\end{array}$ & $\begin{array}{l}325 \\
117\end{array}$ & $\begin{array}{l}4.49 \\
4.49\end{array}$ \\
\hline
\end{tabular}

Abbreviations: BOLD, blood-oxygen-level-dependent; GAD, generalized anxiety disorder; NAC, non-anxious control; PFC, prefrontal cortex; SPM, Statistical Parametric Mapping

Coordinates for peak voxels are presented in the Montreal Neurological Institute (MNI) space. Anatomical mask for the amygdala (left and right) created using SPM's Anatomy toolbox and small volume corrected at $P<0.05$. All other findings are from unrestricted whole-brain analysis with map-wise error rate $P<0.05$. Cluster extents reported for post-registration voxels $\left(2 \mathrm{~mm}^{3}\right)$. 
amygdala activity led to increased RT bias only to the extent that right DLPFC recruitment was deficient.

\section{Discussion}

As predicted, the eStroop elicited opposing patterns of behavior in late-life GAD patients and age-matched NACs, with negative words eliciting task interference in the GAD group while facilitating task performance in the NAC group. These findings support the existence of an attentional bias in late-life GAD similar to that seen in younger GAD patients performing the eStroop. ${ }^{23}$ The neural substrates of this eStroop effect were most consistent with a top-down deficits model of attentional bias. Negative words elicited increased activity in PFC control regions (DMPFC, left ventrolateral PFC) in the NAC group only, consistent with previous findings from non-anxious younger adults. ${ }^{36}$ The GAD group not only failed to match this pattern of PFC recruitment but in fact also showed decreased activity during negative words in bilateral DLPFC regions (Table 3; Figure 1), suggesting a deficit in the ability to implement top-down attentional control in the face of negative emotional distracters. These opposing patterns of PFC recruitment across the two groups were also evident in a direct between-groups comparison, which showed reduced recruitment of the bilateral DLPFC in the GAD group as compared with NACs (Table 2; Figure 1). In the left DLPFC in particular, betweengroup differences were jointly explained by increases to negative words in the NAC group and decreases to negative words in the GAD group, suggesting a pronounced difference in the way this region responds to negative information in the context of clinical anxiety.

The PFC regions that were increased during negative words in the NAC group (DMPFC, ventrolateral PFC) or decreased during negative words in the GAD group (DLPFC) have each been widely implicated in top-down control over stimulus processing, ${ }^{37-39}$ and specifically in studies of top-down negative emotion reduction, ${ }^{40}$ including studies of explicit cognitive reappraisal, ${ }^{41,42}$ as well as more implicit forms of emotion regulation such as affect labeling ${ }^{43}$ and mindfulness meditation. ${ }^{44}$ Thus, these findings imply a deficit in such inhibitory functions in late-life GAD.

It is notable that PFC hypoactivity was seen in the current context, whereas PFC hyperactivity has characterized GAD during prolonged task states such as worry ${ }^{45}$ and rest. ${ }^{46}$ The eStroop task involves an explicit goal of identifying word color as quickly as possible, whereas other goals that subserve this aim, such as rapid inhibition of emotional responses, are likely to be implicitly (non-consciously) held. Consistent with our findings, previous studies assessing implicit emotion regulation during early stages of stimulus processing (including one previous study of the eStroop) have shown a pattern of PFC deficits in samples of younger adult GAD patients ${ }^{47}$ and college undergraduates with high scores on a worry questionnaire. ${ }^{48}$ Thus, implicit top-down emotion regulation during the first several seconds of stimulus processing may be impaired in GAD across the lifespan, necessitating the use of compensatory regulatory strategies during later, prolonged and/or explicit stages of processing. It is also notable that GAD participants' performance on the Stroop Color-Word task, ${ }^{29}$ a test of non-emotional inhibitory control, was equivalent to that of NACs (Table 1), suggesting that inhibitory deficits are not a generalized feature of late-life GAD, but emerge specifically when negative emotional content competes for processing resources.

There were no significant findings in an additional PFC region expected to be relevant to eStroop performance, the ACC, a region implicated in attention and in emotion regulation. ${ }^{49}$ ACC activation has been elicited by emotional interference in some ${ }^{50-52}$ but not all ${ }^{36,53,54}$ previous eStroop studies. In this study, the block design or relatively long duration of the task may have contributed to the null findings, given that ACC activation degrades with practice ${ }^{55}$ and when distracting information is presented frequently (for example, in a block). ${ }^{56}$

Amygdala findings. In comparison with the NAC group, the GAD group showed a larger response to negative words (relative to neutral) in the left amygdala (Table 2). However, this group difference was driven by the NAC group, who showed decreases in the left basolateral amygdala and left hippocampus during negative words (Table 3), rather than by increases in the GAD group. The lack of amygdala increase in the GAD group is contrary to the predictions of a bottom-up model of eStroop interference. It is unlikely that this null finding is due to age-related structural or functional degradation, as previous studies in older adults have shown that the amygdala remains responsive to positive ${ }^{24}$ and novel ${ }^{57}$ stimuli.

No study of adult GAD to date has shown the predicted profile of amygdala hyperresponsivity to negative-vs-neutral material that is a consistent hallmark of many other anxiety disorders, ${ }^{58}$ although some studies have shown a nonspecific increase in amygdala responses to all stimulus conditions. ${ }^{47,59}$ Thus, available neuroimaging findings in GAD point to a unique neurocognitive profile in which PFC mechanisms may have a larger or more singular role than in other forms of clinical anxiety. Consistent with prominent cognitive models of $\mathrm{GAD},{ }^{60}$ prefrontal phenomena such as worry and aberrant top-down attentional control may define the disorder more reliably than do hyperreactive emotional and physiological responses.

In the NAC group, the decreased activity observed in the left amygdala also extended into portions of the left hippocampus, a region associated with contextual and declarative memory that has reciprocal connections to the amygdala. ${ }^{11,61}$ Previous neuroimaging studies have suggested that the ability to inhibit emotional episodic memories is associated with frontal inhibition of the hippocampus and amygdala. ${ }^{62,63}$ Thus, the pattern of increases and decreases observed in the NAC group, in conjunction with NAC behavioral findings, is potentially consistent with the 'positivity effect' literature. NAC participants seemed to inhibit sustained emotional processing of negative material and, potentially, related episodic memories, which were irrelevant to the task at hand (color identification) and had the potential to negatively impact mood.

Correlation and mediation findings. Correlation and mediation analyses were used to explore functional connectivity in the PFC-amygdala circuit. Consistent with a top-down deficits model, the degree to which PFC control 
regions were successfully brought online seems to have been an important determinant of how quickly coloridentification responses were made in the context of emotional distraction. Negative $>$ neutral contrast values in numerous PFC regions were negatively correlated with RT bias scores across all participants, and one PFC region in particular (the right DLPFC) also exhibited an inverse functional connection with the left amygdala (Figure 2), providing three interrelated variables for mediation analysis. Although contrast values from the left amygdala were positively related to bias, this relationship was mediated by right DLPFC decreases, suggesting that amygdala increases led to increased RT bias only in the context of deficient DLPFC recruitment. Although no direct anatomical connections link the DLPFC and amygdala, previous work has suggested the DLPFC nevertheless has an important role in cognitive control of emotion. ${ }^{14,42,64}$ Thus, top-down control mechanisms once again appeared central to the emergence of RT bias in the present sample.

Limitations. As study hypotheses pertained to the effects of anxiety rather than to the effects of aging, no younger adult comparison group was included. The age specificity of the present findings therefore requires further investigation. Although sample sizes were small, the detection of predicted effects suggests that the study was adequately powered to test hypotheses, and correlational/mediational analyses made use of the larger, combined sample $(n=28)$. However, the multiple comparisons correction approach we used restricts our ability to detect small clusters of activation ( $n<102$ voxels) within the whole-brain search space. Finally, in mediation analyses, establishing the temporal sequence of neural events based on an indirect, hemodynamic measure (blood-oxygen-level-dependent signal) presents inherent difficulties. Future studies should aim to build on these findings using experimental designs, for instance, by experimentally manipulating the PFC through brain stimulation.

Conclusions. In summary, results of the current investigation suggest that older adults with GAD show an abnormal attentional profile linked to impoverished recruitment of prefrontal attentional control regions, consistent with a topdown model of attentional bias. The presence of top-down deficits in late-life GAD suggests that treatment strategies designed to target top-down mechanisms, such as repeated practice in executive attention skills ${ }^{65}$ or 'attention training' strategies that aim to build a more benign attentional profile through repeated practice, ${ }^{66,67}$ may be fruitful avenues for improving treatment outcomes in this prevalent, disabling, understudied and difficult-to-treat late-life disorder.

\section{Conflict of interest}

The authors declare no conflict of interest.

Acknowledgements. We gratefully acknowledge Dr Tor Wager for his assistance with this work. This research was supported by National Institutes of Health grant F31 MH081468 awarded to the first author; and by a NARSAD Young Investigator Grant, a Busch Biomedical Grant from Rutgers, and an NIH Center for
Clinical and Translational Science Pilot Award from UMDNJ/RWJ Medical University awarded to the third author.

1. Bar-Haim Y, Lamy D, Pergamin L, Bakermans-Kranenburg MJ, van IMH. Threat-related attentional bias in anxious and nonanxious individuals: a meta-analytic study. Psychol Bull 2007; 133: 1-24.

2. Mogg K, Mathews A, Weinman J. Selective processing of threat cues in anxiety states: a replication. Behav Res Ther 1989; 27: 317-323.

3. Fox E, Russo R, Bowles R, Dutton K. Do threatening stimuli draw or hold visual attention in subclinical anxiety? J Exp Psychol Gen 2001; 130: 681-700.

4. Beekman AT, Bremmer MA, Deeg DJ, van Balkom AJ, Smit JH, de Beurs E et al. Anxiety disorders in later life: a report from the Longitudinal Aging Study Amsterdam. Int J Geriatr Psychiatry 1998; 13: 717-726.

5. Fox LS, Knight BG. The effects of anxiety on attentional processes in older adults. Aging Ment Health 2005; 9: 585-593.

6. Price RB, Siegle G, Mohlman J. Emotional Stroop performance in older adults: effects of habitual worry. Am J Geriatr Psychiatry (in press).

7. Lee LO, Knight BG. Attentional bias for threat in older adults: moderation of the positivity bias by trait anxiety and stimulus modality. Psychol Aging 2009; 24: 741-747.

8. Mather M, Carstensen LL. Aging and motivated cognition: the positivity effect in attention and memory. Trends Cogn Sci 2005; 9: 496-502.

9. Scheibe S, Carstensen LL. Emotional aging: recent findings and future trends. J Gerontol $B$ Psychol Sci Soc Sci 2010; 65B: 135-144.

10. Mogg K, Bradley BP. A cognitive-motivational analysis of anxiety. Behav Res Ther 1998; 36: $809-848$.

11. LeDoux JE. Emotion circuits in the brain. Annu Rev Neurosci 2000; 23: 155-184.

12. Zald $\mathrm{DH}$. The human amygdala and the emotional evaluation of sensory stimuli. Brain Res Brain Res Rev 2003; 41: 88-123.

13. Eysenck MW, Derakshan N, Santos R, Calvo MG. Anxiety and cognitive performance: attentional control theory. Emotion 2007; 7: 336-353.

14. Banich MT, Mackiewicz KL, Depue BE, Whitmer AJ, Miller GA, Heller W. Cognitive control mechanisms, emotion and memory: a neural perspective with implications for psychopathology. Neurosci Biobehav Rev 2009; 33: 613-630.

15. Desimone R, Duncan J. Neural mechanisms of selective visual attention. Annu Rev Neurosci 1995; 18: 193-222.

16. Pessoa L, Kastner S, Ungerleider LG. Attentional control of the processing of neural and emotional stimuli. Brain Res Cogn Brain Res 2002; 15: 31-45.

17. Bishop SJ, Jenkins R, Lawrence AD. Neural processing of fearful faces: effects of anxiety are gated by perceptual capacity limitations. Cereb Cortex 2007; 17: 1595-1603.

18. Bishop S, Duncan J, Brett M, Lawrence AD. Prefrontal cortical function and anxiety: controlling attention to threat-related stimuli. Nat Neurosci 2004; 7: 184-188.

19. Bishop SJ, Duncan J, Lawrence AD. State anxiety modulation of the amygdala response to unattended threat-related stimuli. J Neurosci 2004; 24: 10364-10368.

20. Bishop SJ. Neural mechanisms underlying selective attention to threat. Ann N Y Acad Sci 2008; 1129: 141-152.

21. Bishop SJ. Neurocognitive mechanisms of anxiety: an integrative account. Trends Cogn Sci 2007; 11: 307-316.

22. Byers AL, Yaffe K, Covinsky KE, Friedman MB, Bruce ML. High occurrence of mood and anxiety disorders among older adults: The National Comorbidity Survey Replication. Arch Gen Psychiatry 2010; 67: 489-496.

23. Mogg K, Bradley BP. Attentional bias in generalized anxiety disorder versus depressive disorder. Cognitive Ther Res 2005; 29: 29-45.

24. Mather M, Carstensen LL. Aging and attentional biases for emotional faces. Psychol Sci 2003; $14:$ 409-415.

25. Mohlman J, Eldreth DA, Price RB, Staples AM, Hanson C. Prefrontal-limbic connectivity during worry in older adults with generalized anxiety disorder. Unpublished data.

26. First MB, Spitzer RL, Williams JBW, Gibbon M. Structured Clinical Interview for DSM-IV Axis I Disorders - Patient Edition. New York Psychiatric Institute: New York, 1995.

27. Kaplan E, Goodglass H, Wieintraub S. Boston Naming Test. Experimental Edition. Aphasia Research Center, Boston University: Boston, 1976

28. Wechsler D. Wechsler Abbreviated Scale of Intelligence (WASI). Harcourt Assessment: San Antonio, 1999.

29. Trenerry MR, Crosson B, DeBoe J, Leber WR. Stroop Neuropsychological Screening Test Manual. Psychological Assessment Resources: Odessa, FL, 1989.

30. Gotlib IH, McCann CD. Construct accessibility and depression: an examination of cognitive and affective factors. J Pers Soc Psychol 1984; 47: 427-439.

31. Mohlman J, Price RB, Vietri J. Accentuate the Positive, Eliminate the Negative? Performance of Older GAD Patients and Healthy Controls on a Dot Probe Task. Annual Meeting of the Association for Behavioral and Cognitive Therapies: Orlando, FL, 2008.

32. Ashburner J. A fast diffeomorphic image registration algorithm. Neuroimage 2007; 38: $95-113$

33. Klein A, Andersson J, Ardekani BA, Ashburner J, Avants B, Chiang MC et al. Evaluation of 14 nonlinear deformation algorithms applied to human brain MRI registration. Neuroimage 2009; 46: 786-802.

34. Mechelli A, Henson RN, Price CJ, Friston KJ. Comparing event-related and epoch analysis in blocked design fMRI. Neuroimage 2003; 18: 806-810. 
35. Baron RM, Kenny DA. The moderator-mediator variable distinction in social psychological research: conceptual, strategic, and statistical considerations. J Pers Soc Psychol 1986; 51: 1173-1182.

36. Compton RJ, Banich MT, Mohanty A, Milham MP, Herrington J, Miller GA et al. Paying attention to emotion: an fMRI investigation of cognitive and emotional stroop tasks. Cogn Affect Behav Neurosci 2003; 3: 81-96.

37. Nee DE, Wager TD, Jonides J. Interference resolution: insights from a meta-analysis of neuroimaging tasks. Cogn Affect Behav Neurosci 2007; 7: 1-17.

38. Mansouri FA, Tanaka K, Buckley MJ. Conflict-induced behavioural adjustment: a clue to the executive functions of the prefrontal cortex. Nat Rev Neurosci 2009; 10: 141-152.

39. Venkatraman V, Rosati AG, Taren AA, Huettel SA. Resolving response, decision, and strategic control: evidence for a functional topography in dorsomedial prefrontal cortex. $J$ Neurosci 2009; 29: 13158-13164.

40. Ochsner KN, Gross JJ. The cognitive control of emotion. Trends Cogn Sci 2005; 9 : 242-249.

41. Johnstone $\mathrm{T}$, van Reekum CM, Urry HL, Kalin NH, Davidson RJ. Failure to regulate: counterproductive recruitment of top-down prefrontal-subcortical circuitry in major depression. J Neurosci 2007; 27: 8877-8884.

42. Ochsner KN, Ray RD, Cooper JC, Robertson ER, Chopra S, Gabrieli JD et al. For better or for worse: neural systems supporting the cognitive down- and up-regulation of negative emotion. Neuroimage 2004; 23: 483-499.

43. Lieberman MD, Eisenberger NI, Crockett MJ, Tom SM, Pfeifer JH, Way BM. Putting feelings into words: affect labeling disrupts amygdala activity in response to affective stimuli. Psychol Sci 2007; 18: 421-428.

44. Cahn BR, Polich J. Meditation states and traits: EEG, ERP, and neuroimaging studies. Psychol Bull 2006; 132: 180-211.

45. Paulesu E, Sambugaro E, Torti T, Danelli L, Ferri F, Scialfa G et al. Neural correlates of worry in generalized anxiety disorder and in normal controls: a functional MRI study. Psychol Med 2010; 40: 117-124.

46. Etkin A, Prater KE, Schatzberg AF, Menon V, Greicius MD. Disrupted amygdalar subregion functional connectivity and evidence of a compensatory network in generalized anxiety disorder. Arch Gen Psychiatry 2009; 66: 1361-1372.

47. Etkin A, Prater KE, Hoeft F, Menon V, Schatzberg AF. Failure of anterior cingulate activation and connectivity with the amygdala during implicit regulation of emotional processing in generalized anxiety disorder. Am J Psychiatry 2010; 167: 545-554.

48. Engels AS, Heller W, Mohanty A, Herrington JD, Banich MT, Webb AG et al. Specificity of regional brain activity in anxiety types during emotion processing. Psychophysiology 2007; 44: 352-363.

49. Etkin A, Egner $T$, Kalisch R. Emotional processing in anterior cingulate and medial prefrontal cortex. Trends Cogn Sci 2011; 15: 85-93.

50. Shin LM, Whalen PJ, Pitman RK, Bush G, Macklin ML, Lasko NB et al. An fMRI study of anterior cingulate function in posttraumatic stress disorder. Biol Psychiatry 2001; 50: 932-942.

51. Whalen PJ, Bush G, McNally RJ, Wilhelm S, Mclnerney SC, Jenike MA et al. The emotional counting Stroop paradigm: a functional magnetic resonance imaging probe of the anterior cingulate affective division. Biol Psychiatry 1998; 44: 1219-1228.

52. Britton JC, Gold AL, Deckersbach T, Rauch SL. Functional MRI study of specific animal phobia using an event-related emotional counting Stroop paradigm. Depress Anxiety 2009; 26: $796-805$.
53. Isenberg $\mathrm{N}$, Silbersweig D, Engelien A, Emmerich $\mathrm{S}$, Malavade $\mathrm{K}$, Beattie $\mathrm{B}$ et al Linguistic threat activates the human amygdala. Proc Natl Acad Sci USA 1999; 96: 10456-10459.

54. George MS, Ketter TA, Parekh PI, Rosinsky N, Ring HA, Pazzaglia PJ et al. Blunted left cingulate activation in mood disorder subjects during a response interference task (the Stroop). J Neuropsychiatry Clin Neurosci 1997; 9: 55-63.

55. Milham MP, Banich MT, Claus ED, Cohen NJ. Practice-related effects demonstrate complementary roles of anterior cingulate and prefrontal cortices in attentional control. Neuroimage 2003; 18: 483-493.

56. Milham MP, Banich MT, Barad V. Competition for priority in processing increases prefrontal cortex's involvement in top-down control: an event-related fMRI study of the stroop task. Brain Res Cogn Brain Res 2003; 17: 212-222.

57. Wright $\mathrm{Cl}$, Wedig MM, Williams D, Rauch SL, Albert MS. Novel fearful faces activate the amygdala in healthy young and elderly adults. Neurobiol Aging 2006; 27: 361-374.

58. Etkin A, Wager TD. Functional neuroimaging of anxiety: a meta-analysis of emotional processing in PTSD, social anxiety disorder, and specific phobia. Am J Psychiatry 2007; 164: 1476-1488.

59. Nitschke JB, Sarinopoulos I, Oathes DJ, Johnstone T, Whalen PJ, Davidson RJ et al. Anticipatory activation in the amygdala and anterior cingulate in generalized anxiety disorder and prediction of treatment response. Am J Psychiatry 2009; 166: 302-310.

60. Borkovec TD, Lyonfields JD, Wiser SL, Deihl L. The role of worrisome thinking in the suppression of cardiovascular response to phobic imagery. Behav Res Ther 1993; 31 321-324.

61. Davidson RJ. Affective style, psychopathology, and resilience: brain mechanisms and plasticity. Am Psychol 2000; 55: 1196-1214.

62. Depue BE, Curran T, Banich MT. Prefrontal regions orchestrate suppression of emotional memories via a two-phase process. Science 2007; 317: 215-219.

63. Anderson MC, Ochsner KN, Kuhl B, Cooper J, Robertson E, Gabrieli SW et al. Neural systems underlying the suppression of unwanted memories. Science 2004; 303 232-235.

64. Siegle GJ, Thompson W, Carter CS, Steinhauer SR, Thase ME. Increased amygdala and decreased dorsolateral prefrontal BOLD responses in unipolar depression: related and independent features. Biol Psychiatry 2007; 61: 198-209.

65. Mohlman J. More power to the executive? A preliminary test of CBT plus executive skills training for treatment of late-life GAD. Cogn Behav Pract 2008; 15: 306-316.

66. Amir N, Beard C, Taylor CT, Klumpp H, Elias J, Burns $\mathrm{M}$ et al. Attention training in individuals with generalized social phobia: a randomized controlled trial. J Consult Clin Psychol 2009; 77: 961-973.

67. Hazen RA, Vasey MW, Schmidt NB. Attentional retraining: a randomized clinical trial for pathological worry. J Psychiatr Res 2009; 43: 627-633.

Translational Psychiatry is an open-access journal published by Nature Publishing Group. This work is licensed under the Creative Commons Attribution-NoncommercialNo Derivative Works 3.0 Unported License. To view a copy of this license, visit http://creativecommons.org/licenses/by-nc-nd/3.0/

\section{Supplementary Information accompanies the paper on the Translational Psychiatry website (http://www.nature.com/tp)}

